\title{
THE MASSIVE SATELLITE POPULATION OF MILKY-WAY-SIZED GALAXIES
}

\author{
Aldo Rodríguez-Puebla ${ }^{1}$, Vladimir Avila-ReEse ${ }^{1}$, AND Niv Drory ${ }^{1,2}$ \\ ${ }^{1}$ Instituto de Astronomía, Universidad Nacional Autónoma de México, A. P. 70-264, 04510 México, DF, Mexico; apuebla@astro.unam.mx \\ ${ }^{2}$ McDonald Observatory, The University of Texas at Austin, 1 University Station, Austin, TX 78712-0259, USA \\ Received 2013 April 1; accepted 2013 June 23; published 2013 August 6
}

\begin{abstract}
Several occupational distributions for satellite galaxies more massive than $m_{*} \approx 4 \times 10^{7} M_{\odot}$ around Milky-Way (MW)-sized hosts are presented and used to predict the internal dynamics of these satellites as a function of $m_{*}$. For the analysis, a large galaxy group mock catalog is constructed on the basis of (sub)halo-to-stellar mass relations fully constrained with currently available observations, namely the galaxy stellar mass function decomposed into centrals and satellites, and the two-point correlation functions at different masses. We find that $6.6 \%$ of MW-sized galaxies host two satellites in the mass range of the Small and Large Magellanic Clouds (SMC and LMC, respectively). The probabilities of the MW-sized galaxies having one satellite equal to or larger than the LMC, two satellites equal to or larger than the SMC, or three satellites equal to or larger than Sagittarius (Sgr) are $\approx 0.26,0.14$, and 0.14 , respectively. The cumulative satellite mass function of the MW, $N_{s}\left(\geqslant m_{*}\right)$, down to the mass of the Fornax dwarf is within the $1 \sigma$ distribution of all the MW-sized galaxies. We find that MW-sized hosts with three satellites more massive than Sgr (as the MW) are among the most common cases. However, the most and second most massive satellites in these systems are smaller than the LMC and SMC by roughly 0.7 and $0.8 \mathrm{dex}$, respectively. We conclude that the distribution $N_{s}\left(\geqslant m_{*}\right)$ for MW-sized galaxies is quite broad, the particular case of the MW being of low frequency but not an outlier. The halo mass of MW-sized galaxies correlates only weakly with $N_{s}\left(\geqslant m_{*}\right)$. Then, it is not possible to accurately determine the MW halo mass by means of its $N_{s}\left(\geqslant m_{*}\right)$; from our catalog, we constrain a lower limit of $1.38 \times 10^{12} M_{\odot}$ at the $1 \sigma$ level. Our analysis strongly suggests that the abundance of massive subhalos should agree with the abundance of massive satellites in all MW-sized hosts, i.e., there is not a missing (massive) satellite problem for the $\Lambda \mathrm{CDM}$ cosmology. However, we confirm that the maximum circular velocity, $v_{\max }$, of the subhalos of satellites smaller than $m_{*} \sim 10^{8} M_{\odot}$ is systematically larger than the $v_{\max }$ inferred from current observational studies of the MW bright dwarf satellites; different from previous works, this conclusion is based on an analysis of the overall population of MW-sized galaxies. Some pieces of evidence suggest that the issue could refer only to satellite dwarfs but not to central dwarfs, then environmental processes associated with dwarfs inside host halos combined with supernova-driven core expansion should be on the basis of the lowering of $v_{\max }$.
\end{abstract}

Key words: dark matter - galaxies: dwarf - galaxies: luminosity function, mass function - galaxies: statistics Galaxy: halo - Magellanic Clouds

Online-only material: color figures

\section{INTRODUCTION}

According to the current paradigm of cosmic structure formation and evolution, galaxies form inside cold dark matter (CDM) halos, which grow both by diffuse mass accretion and by incorporation of smaller halos that become subhalos. Inside the subhalos (at least inside the more massive ones) galaxies should also have formed prior to their halo's infall, becoming satellite galaxies. Therefore, the present-day population of satellites around central galaxies is the product of the halo/subhalo assembly and the survival/destruction history of the galaxies inside the subhalos. The $N$-body simulations within the context of the $\Lambda \mathrm{CDM}$ cosmological scenario provide us with the subhalo conditional mass function (subHCMF) as a function of host halo mass $M_{\mathrm{h}}$ (for recent results, see, e.g., Springel et al. 2008; Giocoli et al. 2008; Boylan-Kolchin et al. 2010; Gao et al. 2011; Behroozi et al. 2012). Using this function and statistical models constrained by observations, the central/satellite-halo/subhalo mass connection can be established (e.g., Busha et al. 2011b; Rodríguez-Puebla et al. 2013, hereafter RAD13). In this way, the abundances of the galaxy satellite population as a function of $M_{\mathrm{h}}$ can be calculated (satellite conditional stellar mass function, CSMF). In this paper, our interest is focused on these abundances for systems with a central galaxy of Milky Way (MW) stellar mass, $M_{*, \mathrm{MW}}$.
With the advent of large galaxy surveys, some observational statistical studies of the satellite abundance of central galaxies, in particular those of MW luminosity or mass, have been published. Several statistical distributions have been determined this way, for instance, the fractions of MW-sized galaxies with a given number $N_{s}$ of satellites in the mass range of the Magellanic Clouds (MCs) or with masses equal to or larger than the Large Magellanic Cloud (LMC) or the Small Magellanic Cloud (SMC; James \& Ivory 2011; Tollerud et al. 2011; Liu et al. 2011; Busha et al. 2011a; Robotham et al. 2012). A natural question is whether the $\Lambda \mathrm{CDM}$ scenario makes predictions in agreement with these statistical results related to scales smaller than previously probed.

The works mentioned above conclude that the MW is a rare case with significantly more massive (MC-sized) satellites than other galaxies of similar luminosity or mass. Other studies determine the average luminosity distribution of bright satellites around centrals (Lares et al. 2011; Guo et al. 2011; Wang \& White 2012; Strigari \& Wechsler 2012; Jiang et al. 2012; Sales et al. 2013). The distribution of the MW bright satellites seems to lie above the average found for MW-sized galaxies. In spite of all of these studies, it is not yet clear whether the satellite luminosity (mass) distribution of the MW is rare in a statistically significant sense. It could be that the MW-sized galaxies have a broad range of satellite luminosity distributions, 
the MC-like case being not particularly frequent but not an outlier.

The question on how typical is the MW satellite mass distribution has acquired relevance recently. This distribution, being the best studied one, is compared with subhalo distributions predicted in the context of the $\Lambda \mathrm{CDM}$ and alternative cosmological scenarios in order to test these scenarios at the smallest scales (cf. Boylan-Kolchin et al. 2011b, 2012; Lovell et al. 2012; Vogelsberger et al. 2012; Zavala et al. 2013). However, such a comparison relies (1) on the hope that the MW satellite CSMF is not atypical and (2) on the assumed halo mass for the MW (the subhalo abundance strongly depends on $M_{\mathrm{h}}$, e.g., Gao et al. 2011; Wang et al. 2012). For example, Boylan-Kolchin et al. $(2011 b, 2012)$ have shown that for a few $\Lambda$ CDM halos of $\sim 10^{12} M_{\odot}$ resimulated at very high resolution, there is a significant excess of subhalos with too high masses or maximum circular velocities $\left(v_{\max }>25 \mathrm{~km} \mathrm{~s}^{-1}\right)$ with respect to what is inferred for the MW satellite population (the so-called too big to fail problem). By means of an analytical model for generating a large sample of $\Lambda \mathrm{CDM}$ halos with their corresponding subhalo populations, Purcell \& Zentner (2012) propose that the large variation in the latter among different host halos ameliorates the "too big to fail" problem: at least $\sim 10 \%$ of their MW-sized halos host subhalo populations in agreement with the MW dwarf satellite kinematics. Wang et al. (2012) suggest that the problem is ameliorated if the MW halo mass is simply less massive than it is commonly thought, $M_{\mathrm{h}} \lesssim 10^{12} M_{\odot}$.

In all of these works, the main caveats are the way the MW satellite population is put into the statistical context, and the way the populations of the predicted subhalos and the observed MW satellites are matched. Here, we attempt to overcome these caveats by using a large mock catalog of MW-sized galaxies, constructed on the basis of (sub)halo-to-stellar mass relations fully constrained with currently available observations, namely the galaxy stellar mass function (GSMF) decomposed into centrals and satellites, and the projected two-point correlation functions, 2PCFs, measured at different stellar mass bins (for references see Section 2). While these observations are complete only down to $\approx 2 \times 10^{8} M_{\odot}$, the occupational procedure used to construct the catalog allows one to "extrapolate" observations down to the stellar masses that match the minimum halo/subhalo masses considered here. In RAD13 (see also Busha et al. 2011b), a preliminary attempt to study the massive satellite population of MW-sized galaxies has been presented; however, in that paper the results are given as a function of $M_{\mathrm{h}}$ instead of $M_{*, \mathrm{MW}}$, which introduces the freedom to choose the right $M_{\mathrm{h}}$ to be used for the MW.

Our main result from analyzing the mock catalog is that the $\Lambda$ CDM scenario is statistically consistent with observations regarding the abundances and internal dynamics of satellites in MW-sized galaxies down to satellite stellar masses $m_{*} \sim$ $10^{8} M_{\odot}$. At lower masses, down to the limit of our study $\left(m_{*} \sim 10^{7} M_{\odot}\right)$, the abundances continue being consistent but the internal dynamics of observed dwarf satellites suggest that their subhalos have $v_{\max }$ values smaller than those of the $\Lambda \mathrm{CDM}$ subhalos, under the assumption that the $v_{\max }$ of the latter remain the same after galaxy formation and evolution. Our conclusions are not affected by uncertainties on the matching of subhalo-satellite abundances, on the statistical interpretation of the MW, or on the halo MW mass. Regarding the latter, we instead find the $M_{\mathrm{h}}$ distribution of the MW analogs (see also Busha et al. 2011a).
The layout of this paper is as follows. In Section 2, we briefly describe the semi-empirical occupational approach for linking galaxies to halos and subhalos and how, by using the results of this approach, we construct a mock catalog of 2 million central galaxies, each one with its satellite population down to $m_{*} \sim 10^{7} M_{\odot}$. From this catalog, we select a subsample of about 41,000 central galaxies with MW-like stellar masses. In Section 3, we present different statistical distributions for the massive satellite population of the MW-sized galaxies and compare them to some observational studies. In Section 3.1, we investigate the question of how common the MW satellite mass distribution is, while in Section 3.2 we present the halo mass distribution of the MW analogs. In Section 4, we present $v_{\max }$ versus stellar mass for the mock galaxy (both satellites and centrals) and compare with observations. Our conclusions and a discussion are given in Section 5. We adopt cosmological parameter values close to $W M A P-7: \Omega_{\Lambda}=0.73, \Omega_{\mathrm{M}}=0.27$, $h=0.70, n_{s}=0.98$, and $\sigma_{8}=0.84$.

\section{THE METHOD}

In what follows, we briefly review the semi-empirical approach we use for connecting galaxies to halos and subhalos of different masses. For an extensive presentation of this approach, see Section 2 of RAD13. The approach relies on the assumption that the central-to-halo and satellite-to-subhalo mass relations (CHMR and SSMR, respectively) are monotonic. By parameterizing these mass relations, with their intrinsic scatter included, one can use the predicted $\Lambda \mathrm{CDM}$ distinct halo and conditional subhalo mass functions (HMF and subHCMF) to generate the halo/subhalo occupational distributions for both central and satellite galaxies. Therefore, this method encapsulates the main ideas behind the abundance matching technique, the halo occupation distribution model, and the CSMF formalism (RAD13, see references therein; see also Rodríguez-Puebla et al. 2012). The advantage of the approach is that all the relevant observed statistical distributions of central and satellite galaxies (the GSMF decomposed into centrals and satellites, the CSMFs, and the 2PCFs) are consistently related to each other with the predicted halo/subhalo statistical distributions (the HMF and subHCMF).

The outputs of this approach are the CHMR and SSMR, including their intrinsic scatters, and the satellite CSMFs as a function of halo mass $M_{\mathrm{h}}$. Here we will use the best constrained CHMR and SSMR obtained in RAD13. These relations were (over)constrained by making use of all the available observational information (data set $\mathrm{C}$ in RAD13): the central and satellite GSMFs determined by Yang et al. (2009) down to $2.5 \times 10^{8} M_{\odot}$ and the projected 2PCFs determined by Yang et al. (2012) in five stellar mass bins. For the distinct HMF and subHCMF, the Tinker et al. (2008) and Boylan-Kolchin et al. (2010; see also Gao et al. 2011) fits to cosmological simulations were used, respectively.

\subsection{The Galaxy Group Mock Catalog}

Instead of using the analytical CHMR and SSMR directly, we apply these functions and their scatters to generate a mock galaxy group catalog. With this catalog we will explore several statistical satellite distributions that can be compared with some direct observational determinations given as a function of the central stellar mass. The catalog is generated as follows.

1. From a minimum halo mass of $M_{\mathrm{h}, \min }=10^{10.5} M_{\odot}$, a population of $2 \times 10^{6}$ halos is sampled from the distinct 
HMF. Each halo is randomly picked from this function by generating a random number $U$ uniformly distributed between 0 and 1 and finding the value for $M_{\mathrm{h}}$ that solves the equation $n_{h}\left(M_{\mathrm{h}}\right) / n_{h}\left(M_{\mathrm{h}, \min }\right)=U$. Here $n_{h}$ is the cumulative distinct HMF.

2. To each halo a central galaxy with stellar mass $M_{*}$ is assigned randomly from the probability distribution $P\left(M_{*} \mid M_{\mathrm{h}}\right)$, i.e., the mean $M_{*}-M_{\mathrm{h}}$ relation and its intrinsic scatter which is assumed to be lognormal distributed with a width of 0.173 dex (see RAD13).

3. To each halo defined by its mass $M_{\mathrm{h}}$ a subhalo population above $m_{\text {sub,min }}=10^{9} M_{\odot}$ is assigned randomly by assuming a Poisson distribution (Kravtsov et al. 2004; Boylan-Kolchin et al. 2010). First, the total number of subhalos, $\mathcal{N}_{\text {sub }}$, above $m_{\text {sub,min }}$ is specified by choosing an integer from a Poisson distribution with mean $\left\langle N_{\text {sub }}\left(>m_{\text {sub,min }} \mid M_{\mathrm{h}}\right)\right\rangle$, where this mean number is taken from the subHCMF for the given $M_{\mathrm{h}}$. Then, the mass $m_{\text {sub }}$ for each subhalo is assigned by solving the equation $\left\langle N_{\text {sub }}\left(>m_{\text {sub }} \mid M_{\mathrm{h}}\right)\right\rangle /\left\langle N_{\text {sub }}\left(>m_{\text {sub, } m i n} \mid M_{\mathrm{h}}\right)\right\rangle=u$, where $u$ again is a random number uniformly distributed between 0 and 1 . Note that this last step should be repeated $\mathcal{N}_{\text {sub }}$ times in order to assign subhalo masses to each of the $\mathcal{N}_{\text {sub }}$ subhalos. The lower limit in the subhalo mass is enough to sample satellite galaxies with stellar masses larger than $m_{*} \approx 10^{7} M_{\odot}$ (see Figure 7 of RAD13).

4. To each subhalo we assign a satellite galaxy with stellar mass $m_{*}$, taken from the probability distribution $P\left(m_{*} \mid m_{\text {sub }}\right)$, i.e., the mean $m_{*}-m_{\text {sub }}$ relation and an intrinsic scatter, assumed to be lognormal distributed with a width equal to the central/halo case (the latter assumption seems to be reasonable; see RAD13).

The mock catalog generated in this way reproduces the observational statistical functions used to constrain the CHMR and SSMR, namely the GSMF separated into central and satellite galaxies and the 2PCFs in several mass bins. However, the catalog contains much more information, which can be thought as an "extension" as well as an extrapolation to lower masses of the observations. In particular, we can find the overall satellite number distributions down to $m_{*}=10^{7} M_{\odot}$ around galaxies of a given stellar mass $M_{*}$.

Figure 1 illustrates the mean CHMR (solid line) and its $1 \sigma$ scatter $(0.173 \mathrm{dex}$; gray shaded area) for the data set $\mathrm{C}$ as reported in RAD13. The 2 million mock central galaxies sample this distribution by construction. The short dashed line indicates the mass of central galaxies with $\log M_{*}=$ 10.74, while the dotted lines are 0.1 dex above and below defining a subsample of galaxies with stellar masses in the bin $\log \left(M_{*, \mathrm{MW}} / M_{\odot}\right) \in[10.64,10.84]$, which corresponds to the stellar mass estimate for the MW (Flynn et al. 2006). The 40,694 realizations out of the 2 million that fall within this narrow $M_{*}$ range are represented using black dots (MW-sized galaxies). The shape of the resulting distribution of this subsample of central galaxies as a function of $M_{\mathrm{h}}$ is shown in the bottom panel of the figure. The mean and the standard deviation for this distribution are $\log \left(M_{\mathrm{h}} / M_{\odot}\right)=12.312 \pm 0.277$.

\section{RESULTS AND COMPARISON TO OBSERVATIONS}

In the previous section, we generated a mock catalog of central galaxies corresponding to the stellar mass estimates for the MW. These galaxies have halos in a broad range of masses (see Figure 1). From this sample, we can then establish the

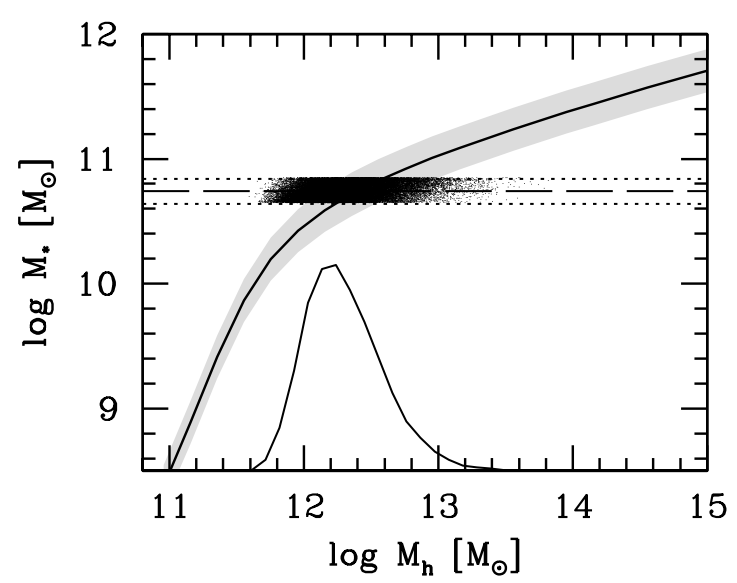

Figure 1. Stellar-to-halo mass relation for central galaxies. The solid line indicates the CHMR reported in RAD13, while the gray shaded area shows the $1 \sigma$ scatter around the mean, assumed to be 0.173 dex. Galaxies that are identified with MW-sized galaxies are those lying in the bin $\log M_{*} \in[10.64,10.84]$ indicated with the dotted lines. The dashed line indicates the mean of this bin. The black dots are the 40,694 realizations of MW-sized galaxies. The resulting distribution as a function of halo mass for MW-sized galaxies is shown below the CHMR. The mean and the standard deviation for this distribution are $\log M_{\mathrm{h}}=12.312$ and 0.277 dex, respectively.

fractions (probabilities) of systems with $N_{s}$ satellites within a (stellar) mass range or above a given mass; this mass cannot be smaller than $m_{*}=10^{7} M_{\odot}$, the minimal mass used to construct the mock catalog (see Section 2.1). Therefore, our results will be restricted to the population of the largest satellites.

For the statistical calculations, we will assume that the stellar masses of the LMC and SMC satellite galaxies are $m_{\mathrm{LMC}}=2.3 \times 10^{9} M_{\odot}$ and $m_{\mathrm{SMC}}=5.3 \times 10^{8} M_{\odot}($ James $\&$ Ivory 2011). We will also consider that the third most massive MW satellite is Sagittarius (Sgr). For a $V$ band, the absolute magnitude of -13.63 mag and a stellar mass-to-luminosity ratio of 2 for $\mathrm{Sgr}^{3}$ imply a stellar mass of $m_{\mathrm{Sgr}}=5 \times 10^{7} M_{\odot}$. Sgr is a tidally stripped dwarf. Based on observations of its tidal tails, a total magnitude (core + tails) of $\approx-15 \mathrm{mag}$ is obtained (Niederste-Ostholt et al. 2010). Then, a rough estimate of the core + tails stellar mass of Sgr is $m_{\mathrm{Sgr}+\mathrm{t}}=1.5 \times 10^{8} \mathrm{M}_{\odot}$. The fourth most massive MW satellite is Fornax (For), with a $V$-band absolute magnitude of $-13.3 \mathrm{mag}$. An estimate of its stellar mass is $m_{\text {For }}=4.3 \times 10^{7} M_{\odot}$ (de Boer et al. 2012).

Our mock catalog was constructed based on observational constraints, so the different satellite population statistics should be consistent with those of real galaxies; we expect that this consistency is preserved for the extrapolations to lower masses using this catalog. In what follows, we compare the results from the mock catalog with observational distributions of MW-sized galaxies and their population of massive satellites. It is important to remark that we do not assume a particular halo mass for the studied MW-sized galaxies.

From a large SDSS sample, Liu et al. (2011) estimate the fraction of $\mathrm{MW}$-sized isolated galaxies that do not have any $\left(N_{\mathrm{MC}}=0\right)$ and that have $N_{\mathrm{MC}}=1,2,3,4,5$, or $6 \mathrm{MC}$-sized satellites. In the same way, we find in our mock catalog the different fractions of MW-sized galaxies with $N_{\mathrm{MC}}=0,1, \ldots, 6$ satellites in the stellar mass range $m_{\mathrm{SMC}}-m_{\mathrm{LMC}}$. Figure 2 shows the predicted probabilities (long-dashed line). The probability of MW-sized galaxies having two MC-sized satellites is

\footnotetext{
3 We assume for Sgr a stellar population with average metallicity $[\mathrm{Fe} / \mathrm{H}] \approx-0.5 \mathrm{dex}$ (Chou et al. 2007; Cole et al. 2005) and average age of 8 Gyr (Bellazzini et al. 2006).
} 


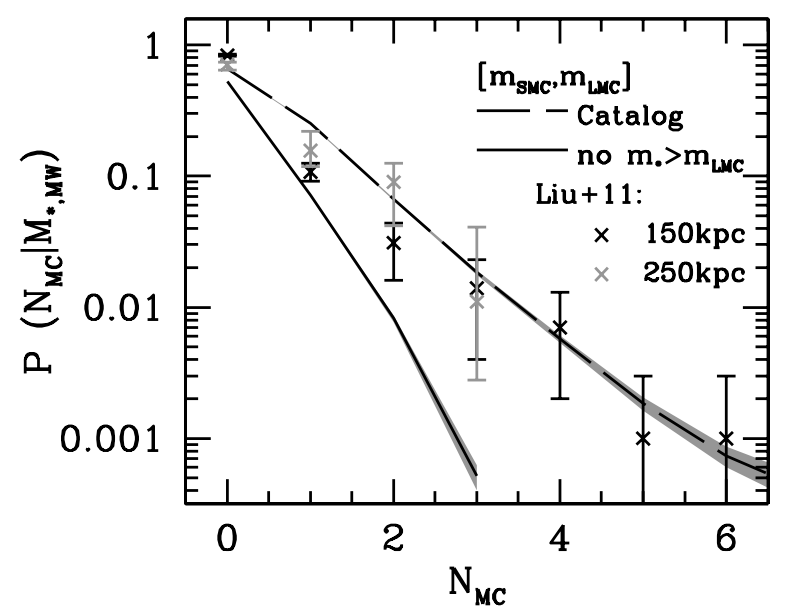

Figure 2. Probability of occurrence of $N_{\mathrm{MC}}$ satellites in the MC mass range around MW-sized galaxies (long-dashed line); the solid line is for the extra condition of no satellites larger than the LMC. The shaded areas are the respective Poissonian errors from the counting. Direct observational results from Liu et al. (2011) are plotted with black (separation from the host up to $150 \mathrm{kpc}$ ) and gray (separations up to $250 \mathrm{kpc}$ ) skeletal symbols.

$6.6 \%$. If, from the subsample of MW-like galaxies having $N_{\mathrm{MC}}$ MC-sized satellites, we exclude those with satellites larger than the LMC, then the probabilities decrease even further (solid line). For $N_{\mathrm{MC}}=2$ and no satellites larger than the LMC, the probability is now only $0.08 \%$. Note that this implies that by far most of those MW-like systems that have $N_{\mathrm{MC}}=2$ should have at least one satellite more massive than the LMC; the MW system does not have such a satellite.

The results from Liu et al. (2011), for a search of MC-sized satellites (not excluding systems with satellites larger than the LMC) up to $150 \mathrm{kpc}$ around the primary, are plotted as crosses in Figure 2. Note that in our case satellites are counted inside the host virial radius $(\sim 200-300 \mathrm{kpc})$. Based on Figure 8 in Liu et al. (2011), we also plot the probabilities when the search radius is increased up to $250 \mathrm{kpc}$ (data are provided only for $N_{\mathrm{MC}}=0,1,2$, and 3 ). It should be said that the selection criteria and observational corrections for searching for MCsized satellites are quite diverse. Liu et al. (2011; see also Busha et al. 2011b) explored the sensitivity of the probabilities to changes in various selection parameters and found that their results can slightly change, with the largest sensitivity being to the satellite search radius around the primary.

The agreement between the probabilities in our mock catalog and the Liu et al. (2011) observations is good within the uncertainties. It is encouraging that the mock catalog predicts the statistics of very rare events, as those systems with $N_{\mathrm{MC}} \geqslant 3$, in good agreement with observations. Regarding the more common events, in the catalog there is a $\sim 66 \%$ chance of MW-sized galaxies without MC-sized satellites, while Liu et al. (2011) report $71 \%$ of such galaxies (for radii up to $250 \mathrm{kpc}$ ); this is because we also have slightly more galaxies with $N_{\mathrm{MC}}=1$ than in Liu et al. (2011; the probabilities of systems with more MC-sized satellites are even lower and do not contribute significantly). These small differences can be explained by the fact that the search radius for satellites in Liu et al. (2011) is up to $250 \mathrm{kpc}$, while in our case there is a fraction of MW-sized galaxies with massive halos, whose virial radii are larger than $250 \mathrm{kpc}$. If the satellite search radius would be increased in Liu et al. (2011), then the fraction of MW-sized galaxies without MC-sized satellites would decrease.

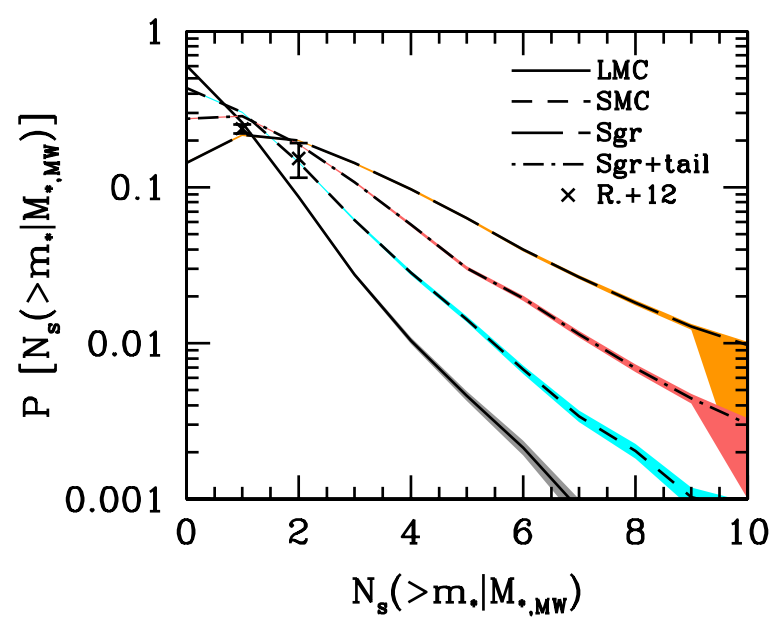

Figure 3. Probability of occurrence of $N_{s}$ satellites around MW-sized galaxies with stellar masses equal to or larger than the LMC, SMC, Sgr+tail, and Sgr (solid, short-dashed, dot-short-dashed, and dashed lines, respectively). The skeletal symbols are the observational inferences by Robotham et al. (2012), corrected for a search radius up to $250 \mathrm{kpc}$, for satellites equal to or more massive than the LMC and the SMC.

(A color version of this figure is available in the online journal.)

An alternative statistical study of MW analog systems was presented in Robotham et al. (2012). Based on a sample of MW-sized galaxies from the new GAMA survey (Driver et al. 2011), they have found the fractions of objects in this sample with one satellite at least as massive as the LMC or with two satellites at least as massive as the SMC. From our mock catalog, we can calculate the fractions of MW-sized galaxies with any number of satellites equal to or larger than a given stellar mass $m_{*}, P\left[N_{s}\left(\geqslant m_{*} \mid M_{*, \mathrm{MW}}\right)\right]$. Figure 3 shows these probabilities for $m_{*} \geqslant m_{\mathrm{LMC}}$ (solid line), $m_{*} \geqslant m_{\mathrm{SMC}}$ (dashed line), and $m_{*} \geqslant m_{\text {Sgr }}$ (long-dashed line; the dot-dashed line is for when the tails of Sgr are included in its mass). The colored contours around the lines are the corresponding Poissonian errors from counting. The probabilities of finding one satellite equal to or more massive than the LMC and two satellites equal to or more massive than the SMC are $26 \%$ and $14.5 \%$, respectively. In the case of Robotham et al. (2012) these probabilities are $11.9 \%$ $(11.2 \%-12.8 \%)$ and $3.4 \%(2.7 \%-4.5 \%)$. However, in Robotham et al. (2012) the satellite search radius was fixed to only $70 \mathrm{kpc}$. From Liu et al. (2011), we roughly estimate the factors by which these fractions could increase if the search radius were to be extended to $250 \mathrm{kpc}$; the factors are at least 2 and 4.5 for $N_{\mathrm{MC}}=1$ and $N_{\mathrm{MC}}=2$, respectively (they could be larger because Liu et al. 2011 limit the search to only satellites in the $m_{\mathrm{LMC}}-m_{\mathrm{SMC}}$ mass range). Taking these correction factors into account, the agreement between the predicted probabilities and those determined by Robotham et al. (2012) becomes quite good.

Recently, several authors have measured the complete (bright) satellite abundances around bright centrals, in particular those with luminosities close to the MW and M31, by using adequate samples from the SDSS (Lares et al. 2011; Guo et al. 2011; Wang \& White 2012; Strigari \& Wechsler 2012; Sales et al. 2013) and from the Canada-France-Hawai Telescope Legacy Survey (Jiang et al. 2012). In each one of these studies, different criteria for the sample selection, different searching and correction methodologies, various radii for the satellite search, etc., were applied. Therefore, the results are not easy to compare.

In general, these works find that the conditional bright satellite luminosity function of $\mathrm{MW} / \mathrm{M} 31$-sized galaxies is described 
by a relatively steep power law, and a normalization such that down to $\sim 6$ mag fainter than the central there is on average a factor of 1.5-2 fewer satellites than the average of the MW and M31. The MW satellite CSMF measured in our mock catalog agrees in general with the above-mentioned studies, but it seems to be slightly overabundant above the mass (or luminosity) corresponding to the SMC, in particular with respect to Wang $\&$ White (2012) and Strigari \& Wechsler (2012). In the case of Jiang et al. (2012, and in a less extent for Guo et al. 2011), a slight flattening at the high end of the luminosity function is seen, which is similar to our case. We recall that the direct observational searches of satellites are for a fixed radius around the central, which is 250 or $300 \mathrm{kpc}$ typically (the exception is Jiang et al. 2012 who use the virial radius determined by the Yang et al. 2007 group finding algorithm). In the mock catalog, we count the satellites inside the virial radius, which for a nonnegligible fraction of galaxies, is larger than $300 \mathrm{kpc}$. Therefore, it is expected that the number of satellites counted in the direct observational studies (especially the most massive ones, which are more likely to be at larger radii) should be slightly lower than in our mock catalog.

We conclude that the population of the largest satellites around MW-sized central galaxies in our mock catalog agrees in general with several direct observational determinations, which present different and limited satellite population statistics. The advantage of our mock catalog, constrained by observations, is that it allows one to calculate any satellite occupational statistics, and to extend the satellite mass limit to masses lower than in current direct observational studies. In this way, one may explore in more detail how the satellite populations of MWsized galaxies are and how particular is the MW system.

\subsection{How Common is the Milky Way System?}

According to Figure 3, the MW is less common than similar sized galaxies in the sense that it has one satellite as massive as the LMC or two satellites equal to or more massive than the SMC; there are more MW-sized galaxies that do not have satellites of mass $m_{*} \geqslant m_{\mathrm{LMC}}(60.6 \%$ versus $26.1 \%$ for those with one satellite) or have less than two satellites more massive than $m_{\mathrm{SMC}}(85.5 \%$ versus $14.5 \%$ for those with two satellites). However, the MW can be considered a common galaxy in the sense that it has three satellites more massive than $m_{\mathrm{Sgr}}$. In general, what we learn from Figure 3 is that the satellite number distributions are relatively wide and there is not a strongly preferred number of satellites above a given mass. For example, the probabilities of having $0,1,2,3$, or 4 satellites with $m_{*} \geqslant m_{\text {Sgr }}$ are within a factor of less than two from each other.

The fact that the satellite number distributions of MW-sized galaxies are broad can also be seen in the plot of the cumulative number of satellites above a given mass $m_{*}$ as a function of $m_{*}, N_{s}\left(\geqslant m_{*} \mid M_{*, \mathrm{MW}}\right)$, which is related to the satellite CSMF discussed above. Figure 4 shows the average (solid line) and the $1 \sigma$ scatter (gray shaded area) of $N_{s}\left(\geqslant m_{*} \mid M_{*, \mathrm{MW}}\right.$ ) from the mock catalog. The latter is quite broad. The cyan line corresponds to the MW (the red line is for when the mass of Sgr includes the tidal tails). The MW massive satellite population is within $1 \sigma$ of the number distribution of satellites as a function of mass of all MW-sized galaxies, being above the average by less than a factor of two at the MC satellite masses, and very close to the average regarding its three (four) satellites equal to or more massive than Sgr (For). By means of direct observational determinations Guo et al. (2011), Strigari \& Wechsler (2012), and Jiang et al. (2012)

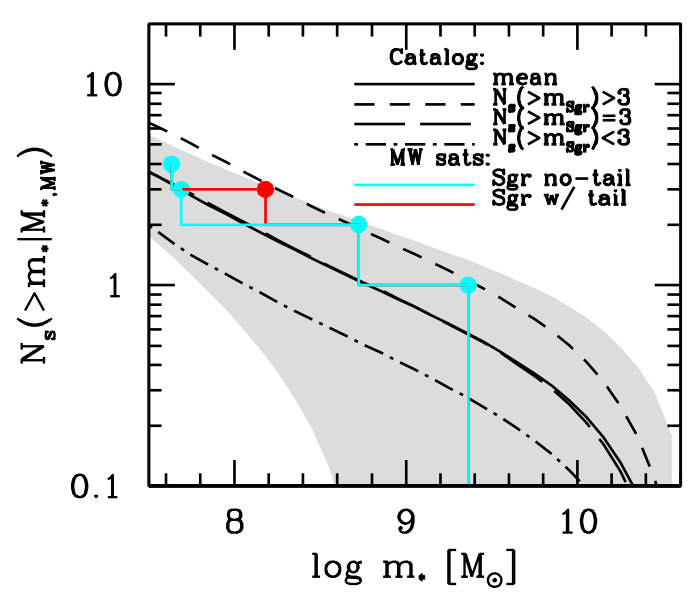

Figure 4. Cumulative satellite mass function of MW-sized galaxies in the mock catalog (solid line) and its $1 \sigma$ scatter (gray shaded area). Subsamples of MW-sized galaxies constrained to have $N_{s} \geqslant 3, N_{s}=3$, and $N_{s} \leqslant 3$ satellites more massive than Sgr are indicated with the short-dashed, long-dashed, and dot-dashed lines, respectively. The cyan staggered line corresponds to the MW satellite galaxies, while the red line is for when the mass of Sgr includes the tidal tails.

(A color version of this figure is available in the online journal.)

arrived at a similar conclusion. From a frequency point of view, we find that the MW-sized galaxies with one satellite $\geqslant m_{\mathrm{LMC}}$ (two satellites $\geqslant m_{\mathrm{SMC}}$ ), as the MW, happen only $1 / 0.6=1.68$ $(2 / 1.02=1.92)$ times less frequently than the average (see also Figure 3).

In fact, given that the (massive) satellite number distribution as a function of mass of MW-sized galaxies is relatively broad, several kinds of "configurations" have close probabilities and all are relatively low. Besides, as more constraints are imposed on the configuration (as for example, to have two satellites in the SMC-LMC mass range but not larger than the LMC; see Figure 2), the lower will be the frequency of occurrence. However, this does not imply that systems with a particular configuration are outliers.

In Figure 4, we also show the mean satellite cumulative mass function of the subsamples of MW-sized galaxies constrained to have $N_{s} \geqslant 3$ (short dashed line), $N_{s}=3$ (long dashed line), and $N_{s} \leqslant 3$ (dot-dashed line) satellites more massive than Sgr. It is interesting to see that galaxies with exactly three satellites more massive than Sgr are close to the average for MW-sized centrals, but they have typically the most and second most massive satellites smaller than the LMC and SMC by roughly 0.7 and 0.8 dex, respectively. The subsample of galaxies with $N_{s} \geqslant 3$ satellites more massive than Sgr better describes the satellite mass function of the MW down to the SMC or to Sgr when including its tails. Finally, we see that the MW definitively does not belong statistically to the subsample of MW-sized centrals with $N_{s} \leqslant 3$ satellites more massive than Sgr, contrary to what is assumed by Wang et al. (2012).

The analysis presented above for the MW system can be applied also to M31. Recent observational results show that M31 has at least twice as many satellites as does the MW (Yniguez et al. 2013). Specifically, it has six satellites brighter than the luminosity of Sgr, making M31 an outlier according to Figure 4. However, the stellar mass of M31 is a factor of 2 larger than the MW (e.g., Tamm et al. 2012). Therefore, it is expected that the M31 halo is more massive than the MW one, hence the M31 halo should host more satellites. This question will be analyzed in detail elsewhere. 


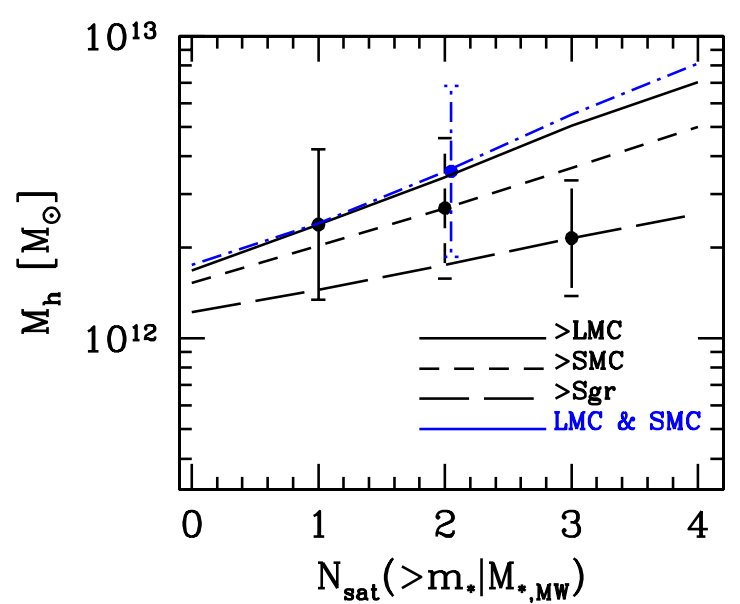

Figure 5. Mean host halo mass of the MW-sized galaxies in the mock catalog as a function of the number of satellites with masses larger than or equal to that of the LMC, SMC, Sgr + tails, and Sgr (see the corresponding lines inside the plot). The case for the interval between the LMC and SMC is also included (blue dot-dashed line). Observe how the halo mass of MW-sized galaxies correlates weakly with the number of satellite galaxies. Nevertheless, from all the cases shown, the MW halo mass is not smaller than $1.38 \times 10^{12} M_{\odot}$ at $1 \sigma$ level.

(A color version of this figure is available in the online journal.)

\subsection{The Halo Masses of MW-like Systems}

The host halo mass distribution of the MW-sized galaxies in the mock catalog is plotted in Figure 1. The distribution is broad, with mean and median values of $2.05 \times 10^{12}$ and $1.91 \times 10^{12} M_{\odot}$. It is known that for clusters and groups of galaxies the total dynamical mass of the system correlates with the richness (number of members above a given mass; see, e.g., Reyes et al. 2008). Is this also the case for MW-like systems? Could we constrain statistically the MW halo mass by its number of satellites above a given mass or within a given mass range?

In Figure 5, we plot the mean host $M_{\mathrm{h}}$ of the mock MW systems with $N_{s}$ satellites with a mass larger than or equal to the LMC (solid line), SMC (short-dashed line), and Sgr (longdashed line), and with masses in between those of the SMC and LMC (dot-dashed blue line). The statistical scatter in all cases is roughly $\sim 0.24 \operatorname{dex}$ in $\log M_{\mathrm{h}}$. For clarity, we plot the scatter (vertical lines) only for the cases corresponding to the MW, i.e., $N_{s}=1$ for the solid line, $N_{s}=2$ for the short-dashed line, $N_{s}=3$ for the long-dashed line, and $N_{s}=2$ for the dot-dashed blue line (slightly shifted horizontally). Figure 5 shows that, in general, there is a correlation of $M_{\mathrm{h}}$ with $N_{s}$ but it is weak. The scatter of $M_{\mathrm{h}}$ around a given $M_{*}$ does not depend significantly on $N_{s}$ for galaxies below the knee in the $M_{*}-M_{\mathrm{h}}$ relation (see Figure 1).

From Figure 5 we can say that at the $1 \sigma$ level, the halo mass of MW-like systems is not smaller than $1.38 \times 10^{12} M_{\odot}$. This limit is for the case of three satellites with $m_{*} \geqslant m_{\text {Sgr }}$ (the mean $M_{\mathrm{h}}$ for this case is $\left.\log \left(M_{\mathrm{h}} / M_{\odot}\right)=12.33\right)$. Interestingly enough, most of the observational estimates of the MW halo mass give values above $10^{12} M_{\odot}$. For example, the most recent work, based on the proper motion of the Leo I dwarf galaxy in combination with numerical simulations, favors a value of $1.6 \times 10^{12} M_{\odot}$ (Boylan-Kolchin et al. 2013 and references therein). For restrictions related to the number of MC-sized satellites, the typical halo masses are slightly larger, as seen in Figure 5; for example, $\log \left(M_{\mathrm{h}} / M_{\odot}\right)=12.430 \pm 0.232$ for the case of two satellites more massive than $m_{\text {SMC }}$. This estimate is somewhat larger than the one obtained by Busha et al. (2011a),

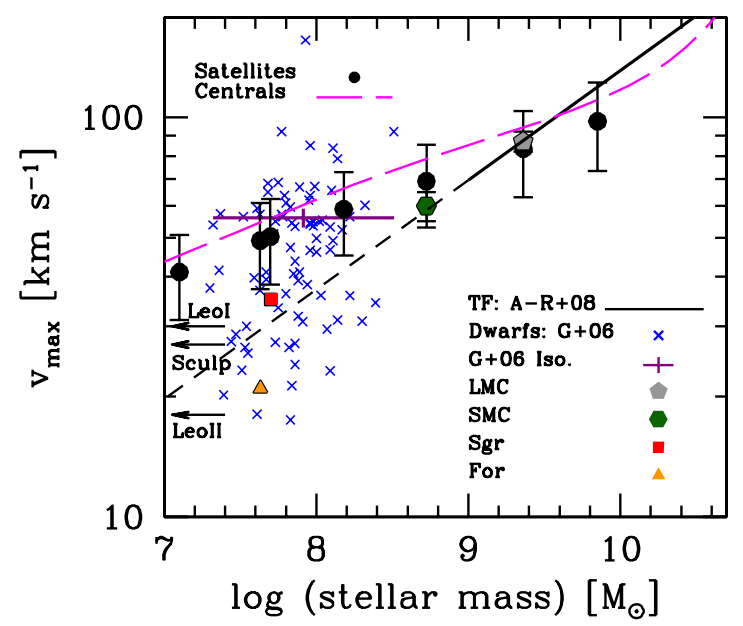

Figure 6. Internal dynamics of dwarf galaxies as a function of stellar mass. Solid circles with error bars show the mean and the standard deviation of the mock catalog subhalo $v_{\max }$ centered at different satellite stellar masses $\left(m_{*}=1.2 \times 10^{7} M_{\odot}, m_{*, \text { For }}, m_{*, \text { Sgr }}, m_{*, \mathrm{SMC}}, m_{*, \mathrm{LMC}}\right.$, and $7.1 \times 10^{9} M_{\odot}$. The magenta long-dashed line indicates the mean $v_{\max }-M_{*}$ relation for the mock central galaxies. Observational estimates for the LMC (Olsen et al. 2011), SMC (Stanimirović et al. 2004), Sgr (Niederste-Ostholt et al. 2010), and For (Strigari et al. 2010) are plotted with the colored filled symbols. The inferred values of $v_{\max }$ by Peñarrubia et al. (2008) for the next three smaller MW dwarfs, Leo I, Sculptor, and Leo II, are indicated with arrows; their stellar masses are smaller than $10^{7} M_{\odot}$. The dashed line is an extrapolation to lower masses of the stellar (inverse) TF relation of field disk galaxies reported in Avila-Reese et al. (2008) down to $\sim 10^{9} M_{\odot}$. Individual measurements of $V_{\text {rot }}$ and stellar mass for both central and satellite dwarfs by Geha et al. (2006) are plotted with crosses. For a subsample of isolated dwarfs, Blanton et al. (2008) report a median $V_{\text {rot }}=56 \pm 3 \mathrm{~km} \mathrm{~s}^{-1}$ (violet cross).

(A color version of this figure is available in the online journal.)

who used the Bolshoi $N$-body cosmological simulation (Klypin et al. 2011) to look for MW-sized halos with two subhalos with maximum circular velocity, $v_{\max }$, larger than $50 \mathrm{~km} \mathrm{~s}^{-1}$ (according to our $v_{\max }-m_{*}$ relation, this mass corresponds to a smaller $m_{*}$ than the one used here for the SMC (see Figure 6), therefore, the host $M_{\mathrm{h}}$ estimated in Busha et al. 2011a would be larger, in better agreement with our study, if they had used the $v_{\max }$ corresponding to $m_{\mathrm{Sgr}}$ ). The orbital information of the MC-sized subhalos in $N$-body simulations has also been used for improving the statistical determinations of the MW halo mass (Boylan-Kolchin et al. 2011a; Busha et al. 2011a), finding that the typical masses should be above $\log \left(M_{\mathrm{h}} / M_{\odot}\right)=12.2-12.3$.

\section{SATELLITE VERSUS $\Lambda$ CDM SUBHALO POPULATIONS}

The statistical method used to construct our mock catalog allows for a connection between satellite and subhalo masses to be made. This connection is constrained by the observed satellite GSMF and the projected correlation functions at different mass bins (see RAD13), and it can be extrapolated to stellar masses lower than the completeness limit of the observational samples. In papers such as Boylan-Kolchin et al. (2011b, 2012) and Lovell et al. (2012), the satellite population of the MW is used to discuss the consistency of the predicted subhalo population in the $\Lambda \mathrm{CDM}$ or $\Lambda \mathrm{WDM}$ scenario, but uncertainty remains about whether the MW and its satellites are a typical system and what the halo mass of the MW is (e.g., Purcell \& Zentner 2012; Wang et al. 2012). With our observationally based catalog, we do not face such a problem since we account for a large population of MW-sized systems (centrals + satellites) with their corresponding host halo masses. 
Our mock catalog offers a statistically complete sample of MW-sized galaxies with their satellite populations, for which we can "measure" the subhalo masses associated with the satellites. By using the tight correlation between the maximum circular velocity, $v_{\max }$, and the mass of the subhalos measured in the Millenium-II Simulation (Boylan-Kolchin et al. 2010; taking into account the scatter around this correlation), the $m_{*}-v_{\max }$ relation and its scatter can be predicted. Note that implicity we are assuming that the subhalo $v_{\max }$ is not altered by baryonic effects. Therefore, in our case, the question is not about consistency between the number of $\Lambda \mathrm{CDM}$ subhalos (above a given $m_{\text {sub }}$ or $v_{\max }$ ) and the number of satellites (above the $m_{*}$ corresponding to $m_{\text {sub }}$ or $v_{\max }$ )-this consistency was established by construction in the method-but about whether the predicted $m_{*}-m_{\text {sub }}$ or $m_{*}-v_{\max }$ relations agree with direct observations.

Figure 6 shows the mean and standard deviation of the $v_{\max }$ versus $m_{*}$ relation for all the satellites above $m_{*}=$ $10^{7} M_{\odot}$ around MW-sized galaxies in our mock catalog. The scatter is due to the dispersions in host halo masses, in the $m_{*}-m_{\text {sub }}$ relation, and in the $m_{*}-v_{\max }$ relation. The dashed line is an extrapolation to lower masses of the stellar (inverse) Tully-Fisher (TF) relation of field disk galaxies as determined from a suitable catalog in Avila-Reese et al. (2008; the stellar mass was shifted by -0.1 dex to convert from the diet-Salpeter to the Chabrier initial mass function). The $v_{\max }-m_{*}$ relation of the satellites seems to bend toward the low-mass side of the TF relation of larger galaxies. In fact, a close trend is followed by central galaxies; the solid line in Figure 6 shows the mean of the $v_{\max }$ versus $M_{*}$ relation for central galaxies in the mock catalog. Such a trend is in agreement with some direct observational studies of the TF relation of dwarf galaxies (cf. McGaugh et al. 2000; De Rijcke et al. 2007; Amorín et al. 2009). The scatter of the $v_{\max }-m_{*}$ (as well as the $v_{\max }-M_{*}$ ) relation increases with decreasing mass, also in agreement with direct observational studies. The bend of the stellar TF relation at velocities below $\sim 100 \mathrm{~km} \mathrm{~s}^{-1}$ and the increase of its scatter is also observed in cosmological numerical simulations (de Rossi et al. 2010) and it is explained by the strong loss of baryons due to supernovadriven feedback in low-amplitude gravitational potentials.

In Figure 6, we also plot the individual measurements of the maximum rotation velocity $V_{\text {rot }}$ and stellar mass for (central and satellite) dwarf galaxies by Geha et al. (2006; crosses). ${ }^{4}$ The scatter is high, and down to stellar masses $\sim 10^{8} M_{\odot}$ most of dwarfs are close to those from our catalog and above the extrapolated TF relation. There are some hints that those dwarfs in the high- $V_{\text {rot }}$ side in the Geha et al. (2006) sample tend to be centrals. For example, Blanton et al. (2008) select the subsample of very isolated dwarfs from Geha et al. (2006); these are certainly central galaxies. They report a median $V_{\text {rot }}=56 \pm 3 \mathrm{~km} \mathrm{~s}^{-1}$ for this subsample, which spans almost the entire mass range of the total sample. This value is also plotted in Figure 6 (violet cross) and it agrees well with the velocities of our central galaxy sample.

For masses smaller than $10^{8} M_{\odot}$, there is a significant fraction of observed dwarfs with lower $v_{\max }$ than the mock dwarfs, although the scatter is high. We also plot the values of $v_{\max }$ and $m_{*}$ inferred observationally for the MW satellites LMC,

\footnotetext{
4 Note that (1) in several cases the $\mathrm{H}_{\mathrm{I}}$ line widths used to estimate $V_{\text {rot }}$ underestimate the real maximum velocity that could be at a radius larger than that where gas is observed; and (2) the galaxy+subhalo $v_{\max }$ after baryon matter is included in the numerical simulations may be significantly lower than in the pure dark matter subhalo $v_{\max }$ (see Section 5 for a discussion).
}

SMC, Sgr, and For (colored filled symbols; for the sources see the figure caption), as well as the inferred values of $v_{\max }$ by Peñarrubia et al. (2008) for the next three smaller MW dwarfs, Leo I, Sculptor, and Leo II (indicated with arrows; their stellar masses are certainly smaller than $10^{7} M_{\odot}$ ). While the LMC and SMC fall close to the mock satellites, the observational inferences of $v_{\max }$ for Sgr and For are smaller than the mean of the mock satellites; a similar difference is expected for the next smaller dwarfs, Leo I, Sculptor, and Leo II. Even the lower $1 \sigma$ scatter, given mainly by those systems in low-mass host halos, is higher than the For $v_{\max }$.

For a large sample of galaxies (and not only for the MW galaxy satellites), the results shown above confirm a potential problem for the small dwarf galaxies (stellar masses $\lesssim 10^{8} M_{\odot}$ ): they seem to be associated with significantly less concentrated (smaller $v_{\max }$ ) systems than those the $\Lambda \mathrm{CDM}$ scenario predicts (Boylan-Kolchin et al. 2011b, 2012). However, the question that remains open is whether this problem refers to both central and satellite galaxies or only to the latter. According to the above, it could be that those dwarfs in the Geha et al. (2006) sample that are in the low- $V_{\text {rot }}$ side are satellites, while those in the high- $V_{\text {rot }}$ side are centrals, and as can be appreciated in Figure 6, they are consistent with the mock central dwarfs.

\section{SUMMARY AND DISCUSSION}

By means of a statistical approach that observationally constrains the galaxy-(sub)halo connection for central and satellite galaxies, we generate a realization of $2 \times 10^{6}$ central galaxies and their populations of satellites. Each galaxy is characterized by its stellar and (sub)halo mass and, by construction, the catalog reproduces (1) the observed central/satellite GSMFs and projected two-point correlation functions in several stellar mass bins down to their completeness limits $\left(m_{*} \sim 2.5 \times 10^{8} \mathrm{M}_{\odot}\right.$, though we extrapolate it down to $\sim 10^{7} M_{\odot}$ ); (2) the $\Lambda \mathrm{CDM}$ distinct halo mass function (Tinker et al. 2008) as well as the subHCMF (Boylan-Kolchin et al. 2010). From this catalog we picked all the central galaxies with MW stellar mass, $\log M_{*}=10.74 \pm 0.1 \mathrm{dex}$ (40,694 objects), and studied the (massive) satellite occupational distributions of these galaxies. The main results from the "observation" of the MW-like systems in the mock catalog are as follows.

1. The fractions (probabilities) of MW centrals with $N_{\mathrm{MC}}$ satellites within the MCs stellar mass range or above the SMC or LMC masses are in general agreement with direct observational studies (Liu et al. 2011; Busha et al. 2011b; Robotham et al. 2012) after correcting for the satellite search radius, which in our case is the virial radius of the host halo (see Figures 2 and 3). For example, we find that the probability of finding two satellites in the $\mathrm{MC}$ mass range is $\sim 6.6 \%$ (or $\sim 0.08 \%$ if we add the condition of having no satellites more massive than the LMC); the probabilities of having one satellite with $m_{*} \geqslant m_{\mathrm{LMC}}$, two satellites with $m_{*} \geqslant m_{\mathrm{SMC}}$, or three with $m_{*} \geqslant m_{\mathrm{Sgr}}$ are $26.1 \%, 14.5 \%$, and $14.3 \%$, respectively. We also find that the average (massive) satellite mass function of the mock MW-sized galaxies is consistent with recent direct observational determinations of the (bright) conditional satellite luminosity function.

2. Having the two most massive satellites be as massive as the MCs makes the MW less common, but it is not a rare case in the sense of an outlier. In our catalog, MW-sized galaxies with one satellite $\geqslant m_{\text {LMC }}$ (two satellites $\geqslant m_{\mathrm{SMC}}$ ), 
as the MW, happen only $1 / 0.6=1.68(2 / 1.02=1.92)$ times less frequently than the average. The cumulative satellite mass function of the MW down to the mass of For is within the $1 \sigma$ distribution of all the MW-sized galaxies, lying above the average by less than a factor of two at the MC masses and close to the average at the For and Sgr masses (Figure 4). MW-sized centrals with exactly three satellites more massive than Sgr are among the most common ones, but they have typically the most and second most massive satellites smaller than the LMC and SMC by roughly 0.7 and 0.8 dex, respectively. In general, we find that the satellite number distributions of MW-sized galaxies are relatively broad.

3. As opposed to clusters and groups of galaxies, the halo mass $M_{\mathrm{h}}$ of $\mathrm{MW}$-sized galaxies correlates weakly with the number of satellites above a given mass (Figure 5). The mean $\log M_{\mathrm{h}}$ and its standard deviation for galaxies with three satellites equal to or more massive than Sgr is $12.33 \pm$ 0.19 . For two satellites with $m_{*} \geqslant m_{\mathrm{SMC}}$ or $m_{*}$ in the SMC-LMC mass range, the mean and standard deviation are $\log M_{\mathrm{h}}=12.430 \pm 0.232$ and $\log M_{\mathrm{h}}=12.552 \pm 0.283$, respectively. Therefore, it is not possible to constrain the halo mass of MW-sized galaxies with appreciable accuracy with the satellite population abundance of the MW, but one can say that at the $1 \sigma$ level, this mass is not smaller than $1.38 \times 10^{12} M_{\odot}$, consistent with recent claims based on the combination of high numerical simulations with the proper motion of Leo I (Boylan-Kolchin et al. 2013).

4. In our catalog of MW-sized galaxies, the number of $\Lambda \mathrm{CDM}$ subhalos (above a given $m_{\text {sub }}$ or $v_{\text {max }}$ ) is consistent with the number of satellites (above the $m_{*}$ corresponding to $m_{\text {sub }}$ or $v_{\max }$ ) by construction and, the satellite abundances of the mock galaxies being in agreement with different direct observational studies, one may conclude that there is not a missing (massive) satellite problem for the $\Lambda \mathrm{CDM}$ model. However, we find an internal dynamics problem: the $v_{\max }$ of the subhalos of satellites smaller than $\sim 10^{8} M_{\odot}$ seems to be systematically larger than the $v_{\max }$ inferred from current observational studies of dwarf satellites, by factors $\sim 1.3-2$ at the masses of Sgr and For (Figure 6). There are some hints that this issue could refer only to the observed satellite dwarfs but not to the central ones.

We conclude that the general agreement of our satellite abundance statistics with direct observations signals toward a self-consistency in the construction of the mock catalog, and it shows that the underlying $\Lambda \mathrm{CDM}$ (sub)halo abundances and internal dynamics are consistent with observations down to the scales of the MC galaxies. For smaller masses, our results point to a possible issue in the internal dynamics of the $\Lambda \mathrm{CDM}$ (sub)halos as compared with the observed satellite dwarfs. These results confirm the conclusions by Boylan-Kolchin et al. (2011b, 2012) for satellite spheroidal dwarfs but with the difference that in our case the results refer to the overall population of MW-sized galaxies. Therefore, our conclusions are free of uncertainties intrinsic to the analysis in Boylan-Kolchin et al. (2011b, 2012) about whether the MW system is atypical or not and for what the MW halo mass is to be used (e.g., Purcell \& Zentner 2012; Wang et al. 2012). However, before arriving at any conclusion, several aspects of these results should be carefully discussed (for an extensive discussion, see Boylan-Kolchin et al. 2012). Here we highlight two observational caveats.

1. Our prediction refers to the maximum circular velocity of the pristine subhalo, $v_{\max }$. Observations refer to the maximum or last-point measured galaxy rotation velocity, $V_{\text {rot }}$, or to a model-dependent $v_{\max }$ constrained by measurements of the stellar velocity dispersion under several assumptions. Because dwarf galaxies are dark matter dominated, in the context of the $\Lambda \mathrm{CDM}$ model it is expected that $v_{\max }$ is attained at a radius much larger than the optical one, where the observational tracers are not available. Then, it could be that the current observational inferences are underestimating the actual values of $v_{\max }$. Regarding the dispersion-supported dwarf spheroidals, their unknown stellar velocity anisotropy and/or halo shape make the inference of their mass distributions ambiguous (e.g., Strigari et al. 2007; Hayashi \& Chiba 2012; Wolf \& Bullock 2012 and references therein).

2. The mock catalog was constructed using both the $m_{*}-m_{\text {sub }}$ and the $M_{*}-M_{\mathrm{h}}$ relations constrained with the Yang et al. (2009) central and satellite GSMFs down to $\sim 2 \times 10^{8}$, as well as with observed projected correlation functions reported in Yang et al. (2012). For smaller masses, we use just an extrapolation of this relation and its scatter. If the satellite GSMF at smaller masses was steeper than the Yang et al. (2009) faint end or the scatter larger than that assigned by us (due, for example, to highly stochastic star formation and tidal effects in the satellite dwarfs), then the relation would be shallower and more scattered, which implies lower subhalo masses (or $v_{\max }$ ) on average at a given $m_{*}$ and higher scatter in these quantities. In Rodríguez-Puebla et al. (2012), by using a low-mass slope of -1.6 for the satellite GSMF, we obtained subhalo masses for $m_{*}=10^{7}-10^{8} M_{\odot}$ dwarfs as small as the tidal masses (close to the subhalo masses) inferred for some MW satellites of these masses. This slope is given by Baldry et al. (2008) for the GSMF, which goes down to $\sim 2.5 \times 10^{7} M_{\odot}$ after applying a correction for surface brightness incompleteness. However, the GSMF in this case refers to all galaxies. In RAD13 we decomposed the Baldry et al. (2008) GSMF into centrals and satellites, resulting then in a $m_{*}-m_{\text {sub }}$ relation that implies subhalo masses larger than the tidal masses by roughly $0.3-0.4$ dex. Future samples, complete down to the smallest masses and decomposed into central and satellite galaxies, should tell us whether or not the satellite GSMF toward very small masses is steep enough to imply subhalo masses (or $v_{\max }$ ) in better agreement with current dynamical studies.

Finally, if the observations regarding the faint end of the satellite GSMF and the internal dynamics of the dwarf satellites remain roughly as those discussed here, then our results could be an indication that the baryonic physics significantly affects the inner structure of the very small subhalos that host dwarf satellites. A possible physical mechanism for explaining the decrease of the inner concentration, and therefore of $v_{\max }$, in low-mass $\Lambda$ CDM (sub)halos could be the feedback-driven gas outflows. By means of $N$-body/Hydrodynamics cosmological simulations, some authors have shown that repeating strong outflows during the halo/galaxy growth are able to drag with them the inner dark matter, producing a decrease of the inner gravitational potential (Mashchenko et al. 2008; Macciò et al. 2012; Governato et al. 2012; Zolotov et al. 2012; Ogiya \& Mori 2012), though it seems difficult that such an effect would be able to lower $v_{\max }$ to the required values (Boylan-Kolchin et al. 2012; di Cintio et al. 2011). However, some numerical simulations show that in the case of satellites, the combination of this effect with the stronger tidal effects of the halo when a central baryonic 
galaxy is included, as well as the lowered baryon fractions of the dwarf satellites, works toward reducing the circular velocities of the simulated MW satellite dwarf spheroidals to the levels required by the results of Boylan-Kolchin et al. (2012) or ours (Brooks \& Zolotov 2012; Arraki et al. 2012; Gritschneder \& Lin 2013).

We have found some hints that the apparent problem of too low circular velocities of dwarfs smaller than $m_{*} \sim 10^{8} M_{\odot}$ refers mostly to satellite galaxies but not to central ones. If this is the case, then such a problem is explained by the plausible physical mechanisms mentioned above. However, if the problem remains for isolated dwarfs, then this could be signaling the necessity of a modification in the cosmological scenario, for example, by introducing warm or self-interacting dark matter (Lovell et al. 2012; Vogelsberger et al. 2012).

We are grateful to Dr. J. Zavala for thoughtful comments on the draft of this paper. We also thank the anonymous referee for a constructive report that helped to improve this paper. A.R-P. acknowledges a graduate student fellowship provided by CONACyT. V.A-R. and N.D. acknowledge CONACyT (ciencia básica) grants 167332 and 128556.

\section{REFERENCES}

Amorín, R., Alfonso, J., Aguerri, J. A. L., Muñoz-Tuñón, C., \& Cairós, L. M. 2009, A\&A, 501, 75

Arraki, K. S., Klypin, A., More, S., \& Trujillo-Gomez, S. 2012, arXiv:1212.6651 Avila-Reese, V., Zavala, J., Firmani, C., \& Hernández-Toledo, H. M. 2008, AJ, 136,1340

Baldry, I. K., Glazebrook, K., \& Driver, S. P. 2008, MNRAS, 388, 945

Behroozi, P. S., Wechsler, R. H., \& Conroy, C. 2013, ApJ, 770, 57

Bellazzini, M., Correnti, M., Ferraro, F. R., Monaco, L., \& Montegriffo, P. 2006, A\&A, 446, L1

Blanton, M. R., Geha, M., \& West, A. A. 2008, ApJ, 682, 861

Boylan-Kolchin, M., Besla, G., \& Hernquist, L. 2011a, MNRAS, 414, 1560

Boylan-Kolchin, M., Bullock, J. S., \& Kaplinghat, M. 2011b, MNRAS, 415, L40

Boylan-Kolchin, M., Bullock, J. S., \& Kaplinghat, M. 2012, MNRAS, 422, 1203

Boylan-Kolchin, M., Bullock, J. S., Sohn, S. T., Besla, G., \& van der Marel, R. P. 2013, ApJ, 768, 140

Boylan-Kolchin, M., Springel, V., White, S. D. M., \& Jenkins, A. 2010, MNRAS, 406, 896

Brooks, A. M., \& Zolotov, A. 2012, arXiv:1207.2468

Busha, M. T., Marshall, P. J., Wechsler, R. H., Klypin, A., \& Primack, J. 2011a, ApJ, 743, 40

Busha, M. T., Wechsler, R. H., Behroozi, P. S., et al. 2011b, ApJ, 743, 117

Chou, M.-Y., Majewski, S. R., Cunha, K., et al. 2007, ApJ, 670, 346

Cole, A. A., Tolstoy, E., Gallagher, J. S., III, \& Smecker-Hane, T. A. 2005, AJ, 129,1465

de Boer, T. J. L., Tolstoy, E., Hill, V., et al. 2012, A\&A, 544, A73

De Rijcke, S., Zeilinger, W. W., Hau, G. K. T., Prugniel, P., \& Dejonghe, H. 2007, ApJ, 659, 1172

de Rossi, M. E., Tissera, P. B., \& Pedrosa, S. E. 2010, A\&A, 519, A89

di Cintio, A., Knebe, A., Libeskind, N. I., et al. 2011, MNRAS, 417, L74
Driver, S. P., Hill, D. T., Kelvin, L. S., et al. 2011, MNRAS, 413, 971

Flynn, C., Holmberg, J., Portinari, L., Fuchs, B., \& Jahreiß, H. 2006, MNRAS, 372,1149

Gao, L., Frenk, C. S., Boylan-Kolchin, M., et al. 2011, MNRAS, 410, 2309

Geha, M., Blanton, M. R., Masjedi, M., \& West, A. A. 2006, ApJ, 653, 240

Giocoli, C., Tormen, G., \& van den Bosch, F. C. 2008, MNRAS, 386, 2135

Governato, F., Zolotov, A., Pontzen, A., et al. 2012, MNRAS, 422, 1231

Gritschneder, M., \& Lin, D. N. C. 2013, ApJ, 765, 38

Guo, Q., Cole, S., Eke, V., \& Frenk, C. 2011, MNRAS, 417, 370

Hayashi, K., \& Chiba, M. 2012, ApJ, 755, 145

James, P. A., \& Ivory, C. F. 2011, MNRAS, 411, 495

Jiang, C. Y., Jing, Y. P., \& Li, C. 2012, ApJ, 760, 16

Klypin, A. A., Trujillo-Gomez, S., \& Primack, J. 2011, ApJ, 740, 102

Kravtsov, A. V., Berlind, A. A., Wechsler, R. H., et al. 2004, ApJ, 609, 35

Lares, M., Lambas, D. G., \& Domínguez, M. J. 2011, AJ, 142, 13

Liu, L., Gerke, B. F., Wechsler, R. H., Behroozi, P. S., \& Busha, M. T. 2011, ApJ, 733,62

Lovell, M. R., Eke, V., Frenk, C. S., et al. 2012, MNRAS, 420, 2318

Macciò, A. V., Stinson, G., Brook, C. B., et al. 2012, ApJL, 744, L9

Mashchenko, S., Wadsley, J., \& Couchman, H. M. P. 2008, Sci, 319, 174

McGaugh, S. S., Schombert, J. M., Bothun, G. D., \& de Blok, W. J. G. 2000, ApJL, 533, L99

Niederste-Ostholt, M., Belokurov, V., Evans, N. W., \& Peñarrubia, J. 2010, ApJ, 712,516

Ogiya, G., \& Mori, M. 2012, arXiv:1206.5412

Olsen, K. A. G., Zaritsky, D., Blum, R. D., Boyer, M. L., \& Gordon, K. D. 2011, ApJ, 737, 29

Peñarrubia, J., McConnachie, A. W., \& Navarro, J. F. 2008, ApJ, 672, 904

Purcell, C. W., \& Zentner, A. R. 2012, JCAP, 12, 7

Reyes, R., Mandelbaum, R., Hirata, C., Bahcall, N., \& Seljak, U. 2008, MNRAS, 390,1157

Robotham, A. S. G., Baldry, I. K., Bland-Hawthorn, J., et al. 2012, MNRAS, 424, 1448

Rodríguez-Puebla, A., Avila-Reese, V., \& Drory, N. 2013, ApJ, 767, 92

Rodríguez-Puebla, A., Drory, N., \& Avila-Reese, V. 2012, ApJ, 756, 2

Sales, L. V., Wang, W., White, S. D. M., \& Navarro, J. F. 2013, MNRAS, 428,573

Springel, V., Wang, J., Vogelsberger, M., et al. 2008, MNRAS, 391, 1685

Stanimirović, S., Staveley-Smith, L., \& Jones, P. A. 2004, ApJ, 604, 176

Strigari, L. E., Bullock, J. S., Kaplinghat, M., et al. 2007, ApJ, 669, 676

Strigari, L. E., Frenk, C. S., \& White, S. D. M. 2010, MNRAS, 408, 2364

Strigari, L. E., \& Wechsler, R. H. 2012, ApJ, 749, 75

Tamm, A., Tempel, E., Tenjes, P., Tihhonova, O., \& Tuvikene, T. 2012, A\&A, 546, A4

Tinker, J., Kravtsov, A. V., Klypin, A., et al. 2008, ApJ, 688, 709

Tollerud, E. J., Boylan-Kolchin, M., Barton, E. J., Bullock, J. S., \& Trinh, C. Q. 2011, ApJ, 738, 102

Vogelsberger, M., Zavala, J., \& Loeb, A. 2012, MNRAS, 423, 3740

Wang, J., Frenk, C. S., Navarro, J. F., Gao, L., \& Sawala, T. 2012, MNRAS, 424,2715

Wang, W., \& White, S. D. M. 2012, MNRAS, 424, 2574

Wolf, J., \& Bullock, J. S. 2012, arXiv: 1203.4240

Yang, X., Mo, H. J., \& van den Bosch, F. C. 2009, ApJ, 695, 900

Yang, X., Mo, H. J., van den Bosch, F. C., et al. 2007, ApJ, 671, 153

Yang, X., Mo, H. J., van den Bosch, F. C., Zhang, Y., \& Han, J. 2012, ApJ, 752,41

Yniguez, B., Garrison-Kimmel, S., Boylan-Kolchin, M., \& Bullock, J. S. 2013, arXiv: 1305.0560

Zavala, J., Vogelsberger, M., \& Walker, M. G. 2013, MNRAS, 431, L20

Zolotov, A., Brooks, A. M., Willman, B., et al. 2012, ApJ, 761, 71 